\title{
The Differences Between Action of Inhaled Silica and Iron Nanoparticles in ApoE-knockout mice - Silica Nanoparticles Exacerbate Atherosclerosis Through Skewing Macrophage Polarization Towards M1 Phenotype
}

\section{Kamila Stachyra}

Jagiellonian University Medical College Faculty of Medicine: Uniwersytet Jagiellonski Collegium Medicum Wydzial Lekarski https://orcid.org/0000-0003-1817-129X

\section{Anna Wiśniewska}

Jagiellonian University Medical College Faculty of Medicine: Uniwersytet Jagiellonski Collegium Medicum Wydzial Lekarski

\section{Anna Kiepura}

Jagiellonian University Medical College Faculty of Medicine: Uniwersytet Jagiellonski Collegium Medicum Wydzial Lekarski

\section{Katarzyna Kuś}

Jagiellonian University Medical College Faculty of Medicine: Uniwersytet Jagiellonski Collegium Medicum Wydzial Lekarski

\section{Filip Rolski}

Jagiellonian University Medical College Faculty of Medicine: Uniwersytet Jagiellonski Collegium Medicum Wydzial Lekarski

\section{Klaudia Czepiel}

Jagiellonian University Medical College Faculty of Medicine: Uniwersytet Jagiellonski Collegium Medicum Wydzial Lekarski

\section{Łukasz Chmura}

Jagiellonian University Medical College Faculty of Medicine: Uniwersytet Jagiellonski Collegium Medicum Wydzial Lekarski

\section{Grzegorz Majka}

Jagiellonian University Medical College Faculty of Medicine: Uniwersytet Jagiellonski Collegium Medicum Wydzial Lekarski

\section{Marcin Surmiak}

Jagiellonian University Medical College Faculty of Medicine: Uniwersytet Jagiellonski Collegium Medicum Wydzial Lekarski

\section{Maciej Suski}


Jagiellonian University Medical College Faculty of Medicine: Uniwersytet Jagiellonski Collegium Medicum Wydzial Lekarski

Rafał Olszanecki ( $\square$ rafal.olszanecki@uj.edu.pl )

https://orcid.org/0000-0001-6966-3732

Research

Keywords: atherosclerosis, nanoparticles, atherosclerotic plaques, apoE-knockout mice, macrophages

Posted Date: April 6th, 2021

DOl: https://doi.org/10.21203/rs.3.rs-376850/v1

License: (9) This work is licensed under a Creative Commons Attribution 4.0 International License. Read Full License 


\section{Abstract}

Background: Exposure to airborne particulate matter, especially its organic part, is strongly related to the development of cardiovascular disorders including atherosclerosis. The role of inorganic part of air pollution in the pathogenesis of atherosclerosis is still unclear. The aim of this study was to investigate the mechanism of action of two substantial components of inorganic part of airborne particulate matter $\left(\mathrm{SiO}_{2}\right.$ and $\left.\mathrm{Fe}_{2} \mathrm{O}_{3}\right)$ in atherosclerosis. To do this, we used apolipoprotein E-knockout mice exposed to silica (SiNPs) and ferric oxide (FeNPs) nanoparticles in a whole body inhalation chamber for 16 weeks. To our knowledge, this is the first time when whole body exposure system was applied to examine the effects of silica and ferric oxide nanoparticles on atherosclerosis development in mouse model of atherosclerosis.

Results: Inhaled silica nanoparticles augmented the atherosclerotic lesions and increased the percentage of pro-inflammatory M1 macrophages in both the plaque and the peritoneum in apoE $\mathrm{E}^{-1-}$ mice. Exposure to ferric oxide nanoparticles did not enhance atherogenesis process, however, it caused significant changes in the atherosclerotic plaque composition (elevated content of CD68-positive macrophages and enlarged necrotic core accompanied by the decreased level of M1 macrophages). Both silica and iron oxide NPs altered the phenotype of T lymphocytes in the spleen by promoting polarization towards Th17 cells.

Conclusions: Exposure to silica and ferric oxide nanoparticles exerts differential impact on atherosclerosis development. Silica nanoparticles enhance atherosclerosis, which is associated with increased level of M1 macrophages, while ferric oxide nanoparticles alter atherosclerotic plaque composition without influencing the size of the lesions.

\section{Introduction}

It is widely recognized that prolonged exposition to airborne particulate matters (PMs) is linked to multiple health problems, especially in terms of deterioration of the respiratory and cardiovascular systems (1-3). The nanoparticles (NPs) of various silica and ferrum oxides represent robust, inorganic components of PMs (4). Studies point out that inhaled nanoparticles influence various types of cells in lungs including epithelial cells, endothelial cells and macrophages (5-7). The direct cytotoxicity of NPs has been mainly attributed to increase of reactive oxygen species (ROS) generation and cellular damage leading to local and systemic inflammatory response (8). Moreover, it has been shown that small size NPs can translocate through the respiratory tract into the circulation and contribute to the progression of cardiovascular diseases, including atherosclerosis (9).

Atherosclerosis is a chronic inflammatory process ongoing in the arterial wall, with significant involvement of immune system and endothelial dysfunction as one of the early and causative hallmarks (10). Recent studies suggest, that metal oxide NPs can influence both the exaggerated immune cells activation and endothelial function, thus perturb the vasculature homeostasis $(11,12)$. Several possible mechanisms of activation of macrophages by NPs have been recently described, including stimulation of TLR4/MyD88/NF-KB pathway (13), promotion of lipid accumulation via activation of endoplasmic 
reticulum stress (14) or inhibition of autophagy (15). However, many aspects of the influence of NPs on these cells still remain unknown. In atherosclerotic plaques, macrophages reside with a continuum of phenotypic polarization states, characterized, at the extremes, by the classical, "pro-inflammatory" M1-like macrophages induced by lymphocyte type $1 \mathrm{~T}$ helper (Th-1) cytokines and by the "anti-inflammatory" M2like macrophages, induced by Th-2 mediators (16). Although recent studies suggest that PMs and NPs can interact with immune cells and alter macrophages phenotype, it is unclear whether such action could have a significant impact on the development of atherosclerosis and plaque morphology (17).

The aim of present study was to investigate the effect of sub-chronic whole body inhalation of selected oxide nanoparticles on the progression of atherosclerosis in apolipoprotein E-knockout mice (apoE $\mathrm{E}^{-/-}$ mice). In our work we have focused on the impact of nanoparticles of two oxides $-\mathrm{SiO}_{2}$ and $\mathrm{Fe}_{2} \mathrm{O}_{3}$, which are the major components of inorganic part of particulate matter of the environmental air pollutants (4).

\section{Results}

\section{The impact of NPs exposure on lung morphology}

Histological examination of the apoE $\mathrm{E}^{-/-}$mice lungs after 16 weeks of NPs inhalation revealed features of mild chronic inflammatory response in both $\mathrm{SiO}_{2}$ and $\mathrm{Fe}_{2} \mathrm{O}_{3}$-exposed groups, in the form of accumulation of aggregates of inflammatory cells (lymphocytes, plasma cells) along the walls of minor respiratory tracts - in a pattern of pulmonary BALT hyperplasia. There were no other changes in the lung parenchyma of mice treated with silica and ferric oxides (Fig. 1).

\section{The influence of NPs on atherosclerosis}

Inhalation of silica nanoparticles increased the size of atherosclerotic lesions in apoE $\mathrm{E}^{-/-}$mice by nearly $20 \%$, as measured by the cross-section method ( $289403 \pm 14578 \mu \mathrm{m}^{2}$ vs $231609 \pm 18113 \mu \mathrm{m}^{2}$; Fig. 2A, B), while the treatment with ferric oxide nanoparticles did not change the area of atherosclerotic plaques $\left(258989 \pm 12053 \mu \mathrm{m}^{2}\right.$ vs $231609 \pm 18113 \mu \mathrm{m}^{2}$; Fig. 2A, B). FeNPs significantly increased the number of CD68-immunopositive macrophages in atherosclerotic lesions of apoE $\mathrm{E}^{-/-}$mice $(35.8 \% \pm 3.3 \%$ vs $24.9 \% \pm$ $1.4 \%)$, whereas SiNPs had no influence on the macrophages plaque content $(30.1 \% \pm 2.9 \%$ vs $24.9 \% \pm$ $1.4 \%$; Fig. 3A, B). Moreover, both silica and ferric oxide nanoparticles increased the necrotic core area in the lesions, as indicated by hematoxylin-eosin (HE) staining $(6.6 \% \pm 0.9 \%$ and $6.9 \% \pm 0.8 \%$ vs $4.1 \% \pm$ $0.4 \%$; Fig. $2 A, C)$. Inhalation of nanoparticles did not cause significant changes in the number of smooth muscle cells in the plaques (Fig. 3A, C). Furthermore, the exposure to nanoparticles did not change the plasma lipid profile in apoE ${ }^{-/-}$mice (Table 1) nor the plasma levels of the majority of the selected inflammatory markers assayed (Table 2). 
Table 1

Plasma lipids level in control and NPs-treated groups.

\begin{tabular}{|lllll|}
\hline Group & TC [mmol/l] & HDL [mmol/I] & LDL [mmol/l] & TG [mmol/I] \\
\hline Control & $15.81 \pm 1.64$ & $1.13 \pm 0.10$ & $13.67 \pm 1.42$ & $0.96 \pm 0.04$ \\
\hline $\mathrm{SiO}_{2}$ & $17.27 \pm 0.44$ & $0.98 \pm 0.05$ & $14.85 \pm 0.37$ & $0.92 \pm 0.04$ \\
\hline $\mathrm{Fe}_{2} \mathrm{O}_{3}$ & $16.05 \pm 0.54$ & $1.05 \pm 0.05$ & $13.97 \pm 0.48$ & $0.93 \pm 0.08$ \\
\hline $\begin{array}{l}\text { Data presented as mean } \pm \text { SEM; } \mathrm{n}=\text { 8 per group. TC - total cholesterol; HDL - high-density } \\
\text { lipoproteins; LDL - low-density lipoproteins; } \mathrm{TG}-\text { triglycerides. }\end{array}$ &
\end{tabular}

Table 2

Plasma concentrations of selected cytokines in control and NPs-treated groups.

\begin{tabular}{|c|c|c|c|c|}
\hline Group & ICAM-1 [ng/ml] & CCL11 [ng/ml] & CXCL1 [pg/ml] & G-CSF [pg/ml] \\
\hline Control & $49.54 \pm 6.99$ & $0.84 \pm 0.10$ & $224.3 \pm 36.42$ & $38.45 \pm 2.28$ \\
\hline $\mathrm{SiO}_{2}$ & $48.90 \pm 8.82$ & $1.35 \pm 0.16$ * & $280.9 \pm 27.39$ & $34.18 \pm 4.02$ \\
\hline $\mathrm{Fe}_{2} \mathrm{O}_{3}$ & $62.28 \pm 6.66$ & $0.93 \pm 0.11$ & $207.6 \pm 24.45$ & $40.28 \pm 7.48$ \\
\hline
\end{tabular}

\section{The influence of NPs on M1/M2 balance}

We evaluated the phenotype of macrophages in atherosclerotic lesions and peritoneum of apoE $\mathrm{E}^{-/-} \mathrm{mice}^{-}$ by means of immunohistochemistry and flow cytometry, respectively. The content of M1-like macrophages (iNOS ${ }^{+} \mathrm{F} 4 / 80^{+}$cells) increased in both the atherosclerotic plaques $(52.0 \% \pm 2.2 \%$ vs $34.1 \%$ $\pm 3.9 \%)$ and peritoneum $(22.3 \% \pm 2.3 \%$ vs $12.9 \% \pm 1.9 \%)$ in mice exposed to silica nanoparticles as compared to the control group (Fig. 4A, B and 5A, B). Surprisingly, the treatment with ferric oxide led to the depletion of M1-like phenotype in the plaques $(20.2 \% \pm 3.1 \%$ vs $34.1 \% \pm 3.9 \%$; Fig. $4 \mathrm{~A}, \mathrm{~B})$ and had no influence on the content of peritoneal M1 macrophages (16.8\% $\pm 3.0 \%$ vs $12.9 \% \pm 1.9 \%$; Fig. $5 \mathrm{~A}$, B). The treatment with both silica and ferric oxide NPs did not influence the content of M2 cells neither in atherosclerotic plaques nor peritoneal cavities of apoE $\mathrm{E}^{-/-}$mice (Fig. 4A, B and 5A, B). The gene expression analysis of $\mathrm{M} 1$ and $\mathrm{M} 2$ markers in aortas of SiNPs-exposed mice revealed two-fold elevation of mRNA level of iNOS (M1 marker) and significant decrease of expression of arginase-1 mRNA (M2 marker) as compared to the control animals (Fig. 6).

\section{The effect of NPs on splenic T lymphocyte phenotype}

Flow cytometry analysis of CD4 + T lymphocyte subsets in the spleen revealed that the percentage of Th17 cells was significantly higher in the SiNPs - $(1.49 \% \pm 0.37 \%$ vs $0.49 \% \pm 0.02 \%)$ and FeNPs - 
treated groups $(1.97 \% \pm 0.49 \%$ vs $0.49 \% \pm 0.02 \%)$ (Fig. $7 \mathrm{C}$ ). Exposure to nanoparticles did not cause a significant changes in the quantity of other subsets of T lymphocytes (Th1, Th2 and Treg) (Fig. 7C). However, both compounds showed the tendencies to increase Th17/Treg and Th1/Th2 ratios (Fig. 7C). The proportion of CD4 + T lymphocytes to the total lymphocytes pool was not altered regardless the NPs treatment (Fig. 7B).

\section{Discussion}

There is a growing body of evidence regarding the negative impact of air pollution (especially its organic part) on the functioning of the cardiovascular system $(18,19)$. However, the role of inorganic constituents of the particulate matter on the development of different cardiovascular diseases (CVD) including atherosclerosis remains less clear. The main finding of our study is that the two oxides: $\mathrm{SiO}_{2}$ and $\mathrm{Fe}_{2} \mathrm{O}_{3}$ (essential components of the inorganic part of airborne particulate pollutants) influence the atherogenesis and lesions morphology in apoE $\mathrm{E}^{-/-}$mice model in different fashion. Our data indicate, that the macrophages are the common target of NPs pro-atherosclerotic actions, resulting in altered phenotype and activation of these cells (Figs. 4 and 5).

Macrophages are the pivotal cells of innate immunity and key regulators of the inflammatory response, both in terms of the antibacterial action as well as the protection against tissue damage (resolution of inflammation) (20). In general, macrophages can be polarized into two different phenotypes: classically activated type $\mathrm{M} 1$, characterized by high-antigen-presenting capacity and production of pro-inflammatory cytokines, and type M2, which displays anti-inflammatory actions (16). Consequently, M1 macrophages promote adaptive Th1-oriented immune responses against various pathogens, while $\mathrm{M} 2$ cells stimulate Th2 branch of immunity and plays a role in resolution of inflammation and wound healing (21). It is well recognized that $\mathrm{M} 1$ macrophages maintain atherogenic low-grade inflammation in the vascular wall and their increased content in atherosclerotic lesions promotes plaque instability. On the contrary, M2 cells have been found to be associated with atherosclerosis regression (20).

It has been shown in vitro that nanoparticles formed by silica and iron compounds can stimulate macrophages to induce reactive oxygen species (ROS) production, activation of NLRLP3 inflammasome and cytokine release (22-24). What is more, several studies indicated a strong ability of silica NPs (SiNPs) to differentiate macrophages towards M1 phenotype (25-27). Our data are in line with these data and extend the observations of the influence of SiNPs on atherogenesis in apoE $\mathrm{E}^{-/-}$mice model. We evidenced, to our best knowledge for the first time, that the progression of atherosclerosis in apoE $\mathrm{E}^{-/-}$mice exposed to SiNPs was associated with the skewing macrophage polarization in atherosclerotic plaques and peritoneum towards $\mathrm{M} 1$ phenotype, as evidenced by immunohistochemical and molecular measurements (Figs. 4, 5 and 6).

By far only one study examined the influence of SiNPs on the progression of atherosclerosis in $\mathrm{apoE}^{-/-}$ mice (14). In this study, the macrophage phenotype was not investigated, while the acceleratory effect of SiNPs on the progression of atherosclerosis has been attributed to lipid accumulation in macrophages 
caused by endoplasmic reticulum (ER) stress-mediated upregulation of CD36 expression. Indeed, ER stress has been recently linked to the regulation of macrophage differentiation towards M1 phenotype $(28,29)$. It is tempting to hypothesize that ER stress may represent a link between SiNPs action and modulation of macrophage polarization. However, ER stress has been also found to contribute to the alternative activation of these cells towards M2 phenotype, thus the role of ER in regulating macrophage phenotype is more intricate (30), while other mechanisms of the influence of SiNPs on the macrophage phenotype may also be involved in the process. More, it has been demonstrated, that $\mathrm{SiO}_{2}$ could polarize primary macrophages toward the M1 phenotype by up-regulation of CD14, a co-receptor of toll-like receptor 4 (TLR4), which involves NFKB pathway, similarly to bacterial lipopolysaccharide LPS (25).

An interesting mechanism of SiNP's influence on the macrophage phenotype may be the enhancement of mitochondrial dysfunction and deregulation of cell metabolism (31). Our study does not make it possible to verify this assumption. On the other hand, in vitro stimulation of endothelial cells (HMEC-1) by low concentrations of $\mathrm{SiO}_{2}$, but not $\mathrm{Fe}_{2} \mathrm{O}_{3}$ results in mitochondrial damage (our unpublished data). The answer to the question whether any of the abovementioned mechanisms are responsible for the SiNPelicited changes in the macrophage polarization in $\mathrm{apoE}^{-/-}$mice requires further research.

Several in vitro studies have shown toxic effects of various iron compounds on cells involved in atherogenesis, such as endothelial cells, smooth muscles and cells of the immune system (i.a. macrophages and lymphocytes) (32-34). They were mainly attributed to the ability of iron compounds to increase the production of ROS, which is widely recognized as one of the most important mechanisms damaging the vessel wall and contributing the progression of atherosclerotic lesions (35). To our knowledge, this study is the first to test the effect of inhaled administration of iron nanoparticles on the development of atherosclerosis in vivo. We evidenced the differences in the $\mathrm{SiO}_{2}$ - and $\mathrm{Fe}_{2} \mathrm{O}_{3}$-mediated effects in terms of their influence on the development and morphology of atherosclerotic plaques (Figs. 2 and 3). The $\mathrm{SiO}_{2}$ augmented the atherogenesis in the aortas of apoE ${ }^{-/-}$mice (Fig. 2), but did not change the content of macrophages in plaques (Fig. 3). On the other hand, $\mathrm{Fe}_{2} \mathrm{O}_{3}$ did not influence the development of atherosclerotic plaques (Fig. 2), but significantly increased their overall CD68-positive cell content (Fig. 3). Surprisingly, this was associated with decreased proportions of both M1 and M2 cells, however, the latter did not reach the statistical significance (Fig. 4B). One can speculate, that FeNPs induce complex changes in lesion morphology, possibly including the de-differentiation of different cell types within the aortic wall. The literature on the in vivo effect of iron NPs on macrophage polarization is sparse and still inconclusive. In recent in vivo study NPs of ferrumxytol - a carbohydrate-coated, superparamagnetic iron oxide nanoparticle - inhibited tumor growth by inducing M1 macrophage polarization. (36). It therefore seems that the macrophage response to FeNP might largely depend on the experimental model.

In contrast to the effect on M1, our results regarding the effect of NPs on the abundance of M2 macrophages are less conclusive. The decreased level of arginase-1 mRNA in aortas of SiNPs-treated mice might reflect decreased content of M2 macrophages, however, this is neither confirmed by 
histochemical staining in plaques nor by flow cytometry of peritoneal macrophages isolated from SiNPstreated mice. Clearly, the influence of NPs on the macrophage polarization requires verification in further studies.

T lymphocytes were shown to play important role in the early stages of atherosclerosis development. It has been demonstrated that the disruption of the balance between pro-atherogenic T-helper 1 and antiatherogenic T-helper 2 cells, as well as between Th17 and regulatory T lymphocytes (Treg) may contribute to atherosclerosis (21). Previous reports have shown that the exposure to air pollution PMs affects $\mathrm{T}$ cells populations in mice by promoting Th1 and Th17 response (37-39). Moreover, several studies indicate that air pollutants could reduce the level of suppressor regulatory T cells in vivo $(40,41)$. In our setting both silica and iron NPs promoted polarization of T lymphocytes in the spleen towards Th17 cells, thus shifting the balance between Th17 and Treg in favour of activation of inflammatory and immune response (Fig. 7). Both compounds also increased Th1/Th2 ratios, however this effect depended more on decrease of Th2 cells and was less pronounced.

Another difference in the action of SiNPs and FeNPs was revealed by the measurements of the levels of selected cytokines in the plasma. Inhaled administration of SiNPs, but not FeNPs, was associated with a significant increase in plasma levels of the CCL11 (eotaxine). The rise of this eosinophil-specific chemokine may suggest the greater role of these cells in the pulmonary inflammatory response induced by $\mathrm{SiO}_{2}$, as compared to $\mathrm{Fe}_{2} \mathrm{O}_{3}$. Interestingly, it has been shown, that silica nanoparticles in particular may act as adjuvants to enhance allergic diseases in mice (42). Whether and to what extent eosinophils could be involved in atherogenesis enhanced by inhaled SiNPs remains to be tested.

Clearly, our study has a major limitation regarding the NPs delivery system. Although the whole body exposure system, allowing for unrestrained movement of animals during inhalation and reducing their stress during experimental procedures, is one of the best solutions enabling for the consistent environmental exposure to administered materials, it has several drawbacks. The compounds are not delivered directly to the respiratory tract thus nor the estimation of the exact quantity of air inhaled by the individual mice neither the precise determination of the exposure dose is impossible. Moreover, mice exposed to NPs in a chamber may cluster in a group, while part of the airborne material can settle on their hair or cage bottom and ultimately end up in the digestive tract. Collectively, these factors could lead to variation in the amount of aspirated compounds among animals $(43,44)$. To address these issues we expanded the size of the experimental groups, as indicated by the power analysis. Our study can also provide important, but only an indirect indication that inhalation with NPs, via influence on macrophage content and/or phenotype in atherosclerotic plaques may reduce plaque stability and increase the risk of plaque rupture. As the plaques in $\mathrm{apoE}^{-/-}$mice are not prone to the rupture, this model is not relevant for assessment of risk of rupture and association of such complication with inhalation of NPs should be estimated directly in other experimental models.

\section{Conclusions}


We conclude, that inhaled $\mathrm{SiO}_{2}$ and $\mathrm{Fe}_{2} \mathrm{O}_{3}$ nanoparticles show different effects on the development and composition of atherosclerotic lesions in a mouse model of atherosclerosis. The proatherogenic effect of SiNPs was associated with increase percentage of pro-inflammatory M1 macrophages in atherosclerotic plaques. In contrast, FeNPs reduced the plaque M1 macrophages content, however, the number of lesional CD68-positive cells was increased. Finally, both NPs enlarged the necrotic core area and shifted the T lymphocytes differentiation towards Th17 cells. The significance of the observed differences for the risk of atherosclerotic complications, as well as relevance of our findings to the human pathology requires further studies.

\section{Materials And Methods}

\section{Sample preparation}

The amorphous silicon oxide $\left(\mathrm{SiO}_{2}\right)$ nanoparticles were purchased from US Research Nanomaterials, Inc (Houston, TX, USA) in the form of aqueous suspension (25wt\%). The particle size of silica ranged between $5-35 \mathrm{~nm}$. Iron (III) oxide $\left(\mathrm{Fe}_{2} \mathrm{O}_{3}\right)$ was obtained from PlasmaChem (Berlin, Germany) as 5\% nanoparticles aqueous suspension with average particle size 4-8 nm. The stock suspensions of nanoparticles were diluted in distilled water to prepare working solutions for in vivo experiments. Final concentrations of nanoparticles administered to mice were $0.6 \mathrm{mg} / \mathrm{ml}$ and $0.4 \mathrm{mg} / \mathrm{ml}$ for silica nanoparticles (SiNPs) and ferric oxide nanoparticles (FeNPs), respectively. The working solutions were freshly prepared every day.

\section{Animal Experiments}

All animal procedures were in agreement with the guidelines from Directive 2010/63/EU of the European Parliament on the protection of animals used for scientific purposes and were approved by the Jagiellonian University Ethical Committee on Animal Experiments (approval number 105/2018).

Thirty-six female apolipoprotein E-knockout mice on the C57BL/6J background were purchased from Charles River (Wilmington, MA). The animals were housed in a cages with access to feed and water ad libitum and maintained on reversed 12-h dark/12-h light cycles in air conditioned room $\left(22.5 \pm 0.5^{\circ} \mathrm{C}, 50 \pm\right.$ $5 \%$ humidity). After two weeks of acclimatization, at the age of 8 weeks, mice were put on high fat diet containing $10 \%$ fat (Sniff, Germany). The animals were divided into three groups: control $(n=12), \mathrm{SiO}_{2}-$ treated mice $(n=12)$ and $\mathrm{Fe}_{2} \mathrm{O}_{3}$-treated mice $(n=12)$. The aqueous suspensions of silica and ferric oxide nanoparticles were administered by nebulization 5 hours/day, 5 days/week for 16 weeks. The inhalation was performed in a whole body exposition chamber that allowed free animal movement. During the $5 \mathrm{~h}$ daily exposure period the animals were not receiving feed and water. Nebulization was performed by the Aeroneb Pro Nebulizer (Aerogen, Galway, Ireland) directly connected to the chamber. The dose concentration of obtained aerosol was $400 \mu \mathrm{g} / \mathrm{m}^{3}$. The control group was exposed to distilled water under the same conditions as nanoparticles-treated groups. At the end of the treatment period, the mice were injected with 1000 IU of fraxiparine into the peritoneum (Sanofi-Synthelabo, Paris, France) and 
sacrificed in a carbon dioxide chamber. Next, the blood and peritoneal macrophages were collected, after which the selected tissues (heart, aorta, lung and spleen) were dissected.

\section{Histology of the lung}

The lung tissue samples were fixed in $4 \%$ buffered formalin, routinely processed and embedded in paraffin. The paraffin blocks were sectioned and stained with the hematoxylin-eosin method.

\section{Atherosclerosis studies}

The evaluation of atherosclerosis in apoE-knockout mice was performed by cross-section method, according to the previously described protocol (45). The aortic sections (10- $\mu \mathrm{m}$ thickness) were stained with Oil Red-O (Sigma-Aldrich, St. Louis, MO) to assess the areas of atherosclerotic lesions. The necrotic cores in atherosclerotic plaques were evaluated using hematoxylin-eosin (HE) staining. All aortic images were captured by Axio Vert.A1 microscope (Zeiss, Jena, Germany) and data were analysed using the LSM Image Browser software (Zeiss, Jena, Germany).

\section{Immunohistochemistry}

To assess the influence of treatment on atherosclerotic plaque structure and macrophage polarization immunohistochemical analysis of aortic sections was performed. The antibodies against CD68 (dilution 1:600; Bio-Rad, Hercules, CA) and smooth muscle a-actin (SMA) (dilution 1:800; Sigma, St. Louis, MO) were used for total macrophages and smooth muscles detection, respectively. The total area of atherosclerotic plaque occupied by CD68-immunopositive macrophages and smooth muscle cells was measured using the LSM Image Browser software (Zeiss, Jena, Germany). Macrophage polarization in atherosclerotic plaques was examined using antibodies against F4/80 (dilution 1:100; Bio-Rad, Hercules, CA) and nitric oxide synthase 2 (iNOS) (dilution 1:100; Abcam, Cambridge, Great Britain) or arginase 1 (dilution 1:200; Abcam, Cambridge, Great Britain), for detection total macrophages, M1 and M2 phenotype, respectively. Cell nuclei were stained with Hoechst 33342 (Thermo, Waltham, MA). Clear orange signals resulting from superposition of red (iNOS/agninase- 1 ) and green (F4/80) channels and surrounding blue nuclei were considered as positive cells corresponding to macrophage phenotypes, as described before (46). The positive cells detected by co-localization of red (M1/M2 phenotype), green (total macrophages) and blue (cell nuclei) signal were counted and divided by the number of total macrophages. The results were presented as the percent of respective macrophage phenotype. The four immunohistochemical images per phenotype per biological replicate were analysed independently by three examiners and the results were averaged for final quantitation of macrophage phenotypes. All section images were registered using fluorescence microscope with the ZEN software (Zeiss, Jena, Germany).

\section{Biochemical analysis}

The whole blood was centrifuged for 10 minutes at $1000 \times \mathrm{g}$ and $4^{\circ} \mathrm{C}$. Collected plasma was stored at $-80^{\circ} \mathrm{C}$. The plasma levels of total cholesterol, low-density lipoprotein cholesterol (LDL-C), high-density lipoprotein cholesterol (HDL-C) and triglycerides were measured using Cobas 8000 analyser (Roche 
Diagnostics, Indianapolis, IN). In addition, the plasma levels of selected inflammatory markers, such as soluble Intercellular Adhesion Molecule 1 (sICAM-1), Eotaxin-1 (CCL11), Growth-Regulated alpha Protein (CXCL-1) and Granulocyte Colony-Stimulating Factor (G-CSF) were assayed by Luminex Mouse Magnetic Assay (R\&D Systems, NE Minneapolis, US), according to the manufacturer's instruction.

\section{Flow cytometry of peritoneal macrophages}

Macrophages were isolated from the mice by flushing peritoneal cavity with $5 \mathrm{ml}$ PBS and collecting peritoneal fluid. The cells were centrifuged, washed and stained with anti-CD11b (BioLegend, San Diego, CA), anti-F4/80 (BioLegend, San Diego, CA), anti-iNOS (eBioscience/Thermo, Waltham, MA) and antiCD206 (BioLegend, San Diego, CA). For intracellular staining the cells were incubated with BD Cytofix/Cytoperm solution. The measurements were performed using FACSCanto II flow cytometer (Becton Dickinson, Franklin Lakes, NJ) with FACSDiva software.

\section{Flow cytometry of splenocytes}

Spleens were cut into small pieces and disintegrated using $70 \mu \mathrm{m}$ tissue strainers (Corning/Merck, Darmstadt, Germany). The red blood cells were lysed using ACK Lysis Buffer (Gibco/Thermo, Waltham, MA). Then, the cell suspensions were rubbed through $40 \mu \mathrm{m}$ strainers (Falcon, Glendale, AZ), centrifuged and seeded into RPMI-1460 Medium (Sigma, St. Louis, MO), supplemented with 10\% FBS (Gibco/Thermo, Waltham, MA) and penicillin-streptomycin (Sigma, St. Louis, MO). The cells were treated with the Cell Stimulation Cocktail (Thermo, Waltham, MA) for 4 hours in the presence of GolgiStop Protein Transport Inhibitor (Becton Dickinson, Franklin Lakes, NJ). After indicated time, the stimulated cells were harvested, washed and stained using Mouse Th1/Th2/Th17 and Mouse Th17/Treg Phenotyping Kits (Becton Dickinson, Franklin Lakes, NJ), according to the manufacturer's instructions. The fluorescent antibodies specific for mouse CD4, IFN-y (for Th1 detection), IL-4 (for Th2 detection), IL-17A (for Th17 detection) and FoxP3 (for Treg cells detection) were used to perform flow cytometric analysis of the Th cell subtypes. The samples were collected using FACSCanto II analyzer (Becton Dickinson, Franklin Lakes, NJ) and the data were analysed with the FACSDiva software.

\section{Real-time PCR}

Total RNA was isolated from the aorta using the RNeasy Fibrous Tissue Mini Kit (Qiagen, Hilden, Germany), according to the manufacturer's instructions. The RNA concentration was measured at a wavelength of $260 \mathrm{~nm}$ (A260) in an Synergy H1 microplate reader (BioTek Instruments, Inc., Winooski, VT). The purity of obtained total RNA was determined by the A260/A280 ratio. The reverse transcription was done using a High-Capacity Reverse Transcription Kit (Applied Biosystems, Foster City, CA). Real time quantitative PCR was conducted on the Bio-Rad CFX96 Touch $^{\text {TM }}$ Real-Time PCR System with GoTaq ${ }^{\circledR}$ qPCR Master Mix (Promega, Madison, WI). Primers for Actinb and iNOS were obtained from Bio-Rad (Hercules, California, USA). Primers for Arg-1, Mgl-1, Mrc-1, IL-6 and TNFa were purchased from RealTimePrimers.com (Elkins Park, PA). Data analysis was performed by the $2^{-\Delta \Delta C t}$ method. Normalization of the data was performed using Actinb expression.

\section{Statistical analysis}


The statistical analysis was performed using GraphPad Prism 8.3.0. (GraphPad Software Inc., San Diego, $\mathrm{CA}$ ). Results were showed as mean \pm standard error of measurement (SEM). The normality of the data and homogeneity of variance were examined. Then, the statistical significance of differences between the groups was analysed by one-way ANOVA following the Dunnett's multiple comparisons test, or by nonparametric Kruskal-Wallis analysis, when applicable. Values of $p<0.05$ were considered statistically significant.

\section{Declarations}

\section{Declarations}

\section{Ethics approval and consent to participate}

All animal procedures were in agreement with the guidelines from Directive 2010/63/EU of the European Parliament on the protection of animals used for scientific purposes and were approved by the Jagiellonian University Ethical Committee on Animal Experiments (approval number 105/2018).

\section{Consent for publication}

Not applicable

\section{Availability of data and materials}

The datasets used and/or analysed during the current study are available from the corresponding author on reasonable request.

\section{Competing interests}

The authors declare that they have no competing interests.

\section{Funding}

This research was funded by grant from the Polish National Science Centre (Symfonia APARIC 2015/16/W/ST5/00005).

\section{Authors' contributions}

RO and KS were responsible for the conception and design of the study. KS, AW, AK, KK, FR, KC, GM, M. Surmiak and M. Suski performed the experiments. KS, AW, AK, FR and $Ł C$ analysed the data. KS and RO were responsible for writing the manuscript. RO and M. Suski revised the manuscript. All authors read and approved the final manuscript.

\section{Acknowledgements}

Not applicable 


\section{References}

1. Brunekreef B, Holgate ST. Air pollution and health. Lancet Lond Engl. 2002 Oct 19;360(9341):123342.

2. Losacco C, Perillo A. Particulate matter air pollution and respiratory impact on humans and animals. Environ Sci Pollut Res Int. 2018 Dec;25(34):33901-10.

3. Fiordelisi A, Piscitelli P, Trimarco B, Coscioni E, laccarino G, Sorriento D. The mechanisms of air pollution and particulate matter in cardiovascular diseases. Heart Fail Rev. 2017 May;22(3):337-47.

4. Zeb B, Alam K, Sorooshian A, Blaschke T, Ahmad I, Shahid I. On the Morphology and Composition of Particulate Matter in an Urban Environment. Aerosol Air Qual Res. 2018 Sep;28(6):1431-47. 18(.

5. Karlsson HL, Cronholm P, Gustafsson J, Möller L. Copper oxide nanoparticles are highly toxic: a comparison between metal oxide nanoparticles and carbon nanotubes. Chem Res Toxicol. 2008 Sep;21(9):1726-32.

6. Lee K-I, Su C-C, Fang K-M, Wu C-C, Wu C-T, Chen Y-W. Ultrafine silicon dioxide nanoparticles cause lung epithelial cells apoptosis via oxidative stress-activated PI3K/Akt-mediated mitochondria- and endoplasmic reticulum stress-dependent signaling pathways. Sci Rep. 2020 Jun 18;10(1):9928.

7. Tian G, Wang J, Lu Z, Wang H, Zhang W, Ding W, et al. Indirect effect of PM1 on endothelial cells via inducing the release of respiratory inflammatory cytokines. Toxicol Vitro Int J Publ Assoc BIBRA. 2019 Jun;57:203-10.

8. Nemmar A, Holme JA, Rosas I, Schwarze PE, Alfaro-Moreno E. Recent advances in particulate matter and nanoparticle toxicology: a review of the in vivo and in vitro studies. BioMed Res Int. 2013;2013:279371.

9. Engin AB, Neagu M, Golokhvast K, Tsatsakis A. NANOPARTICLES AND ENDOTHELIUM: AN UPDATE ON THE TOXICOLOGICAL INTERACTIONS. 2015;63:13.

10. Wolf D, Ley K. Immunity and Inflammation in Atherosclerosis. Circ Res. 2019 Jan 18;124(2):315-27.

11. Liu X, Sun J. Endothelial cells dysfunction induced by silica nanoparticles through oxidative stress via JNK/P53 and NF-kappaB pathways. Biomaterials. 2010 Nov;31(32):8198-209.

12. Sun J, Wang S, Zhao D, Hun FH, Weng L, Liu H. Cytotoxicity, permeability, and inflammation of metal oxide nanoparticles in human cardiac microvascular endothelial cells: cytotoxicity, permeability, and inflammation of metal oxide nanoparticles. Cell Biol Toxicol. 2011 Oct;27(5):333-42.

13. Geng J, Liu H, Ge P, Hu T, Zhang Y, Zhang X, et al. PM2.5 promotes plaque vulnerability at different stages of atherosclerosis and the formation of foam cells via TLR4/MyD88/NFKB pathway. Ecotoxicol Environ Saf. 2019 Jul 30;176:76-84.

14. Ma R, Qi Y, Zhao X, Li X, Sun X, Niu P, et al. Amorphous silica nanoparticles accelerated atherosclerotic lesion progression in ApoE-/- mice through endoplasmic reticulum stress-mediated CD36 up-regulation in macrophage. Part Fibre Toxicol. 2020 Oct;2(1):50. 17(.

15. Wan Q, Yang M, Liu Z, Wu J. Atmospheric fine particulate matter exposure exacerbates atherosclerosis in apolipoprotein E knockout mice by inhibiting autophagy in macrophages via the 
PI3K/Akt/mTOR signaling pathway. Ecotoxicol Environ Saf. 2021 Jan 15;208:111440.

16. Rojas J, Salazar J, Martínez MS, Palmar J, Bautista J, Chávez-Castillo M, et al. Macrophage Heterogeneity and Plasticity: Impact of Macrophage Biomarkers on Atherosclerosis. Scientifica. 2015;2015:851252.

17. Zhao Q, Chen H, Yang T, Rui W, Liu F, Zhang F, et al. Direct effects of airborne PM2.5 exposure on macrophage polarizations. Biochim Biophys Acta. 2016 Dec;1860(12):2835-43.

18. Combes A, Franchineau G. Fine particle environmental pollution and cardiovascular diseases. Metabolism. 2019 Nov;100S:153944.

19. Meo SA, Suraya F. Effect of environmental air pollution on cardiovascular diseases. Eur Rev Med Pharmacol Sci. 2015 Dec;19(24):4890-7.

20. Shirai T, Hilhorst M, Harrison DG, Goronzy JJ, Weyand CM. Macrophages in vascular inflammationFrom atherosclerosis to vasculitis. Autoimmunity. 2015 May;48(3):139-51.

21. Bartlett B, Ludewick HP, Misra A, Lee S, Dwivedi G. Macrophages and T cells in atherosclerosis: a translational perspective. Am J Physiol-Heart Circ Physiol. 2019 Aug 1;317(2):H375-86.

22. Morishige T, Yoshioka Y, Inakura $H$, Tanabe A, Yao X, Narimatsu S, et al. The effect of surface modification of amorphous silica particles on NLRP3 inflammasome mediated IL-1beta production, ROS production and endosomal rupture. Biomaterials. 2010 Sep;31(26):6833-42.

23. Naqvi S, Samim M, Abdin M, Ahmed FJ, Maitra A, Prashant C, et al. Concentration-dependent toxicity of iron oxide nanoparticles mediated by increased oxidative stress. Int J Nanomedicine. 2010 Nov 16;5:983-9.

24. Du X, Jiang S, Zeng X, Zhang J, Pan K, Song L, et al. Fine particulate matter-induced cardiovascular injury is associated with NLRP3 inflammasome activation in Apo E-/- mice. Ecotoxicol Environ Saf. 2019 Jun 15;174:92-9.

25. Lucarelli M, Gatti AM, Savarino G, Quattroni P, Martinelli L, Monari E, et al. Innate defence functions of macrophages can be biased by nano-sized ceramic and metallic particles. Eur Cytokine Netw. 2004 Dec;15(4):339-46.

26. Park E-J, Park K. Oxidative stress and pro-inflammatory responses induced by silica nanoparticles in vivo and in vitro. Toxicol Lett. 2009 Jan 10;184(1):18-25.

27. Bancos S, Stevens DL, Tyner KM. Effect of silica and gold nanoparticles on macrophage proliferation, activation markers, cytokine production, and phagocytosis in vitro. Int $\mathrm{J}$ Nanomedicine. 2015;10:183-206.

28. Díaz-Bulnes P, Saiz ML, López-Larrea C, Rodríguez RM. Crosstalk Between Hypoxia and ER Stress Response: A Key Regulator of Macrophage Polarization. Front Immunol. 2019;10:2951.

29. Yang F, Liu Y, Ren H, Zhou G, Yuan X, Shi X. ER-stress regulates macrophage polarization through pancreatic EIF-2alpha kinase. Cell Immunol. 2019 Feb;336:40-7.

30. Oh J, Riek AE, Weng S, Petty M, Kim D, Colonna M, et al. Endoplasmic reticulum stress controls M2 macrophage differentiation and foam cell formation. J Biol Chem. 2012 Apr 6;287(15):11629-41. 
31. Saha S, Shalova IN, Biswas SK. Metabolic regulation of macrophage phenotype and function. Immunol Rev. 2017 Nov;280(1):102-11.

32. Zhu M-T, Wang B, Wang $Y$, Yuan L, Wang H-J, Wang M, et al. Endothelial dysfunction and inflammation induced by iron oxide nanoparticle exposure: Risk factors for early atherosclerosis. Toxicol Lett. 2011 Jun 10;203(2):162-71.

33. Ying E, Hwang H-M. In vitro evaluation of the cytotoxicity of iron oxide nanoparticles with different coatings and different sizes in A3 human T lymphocytes. Sci Total Environ. 2010 Sep 15;408(20):4475-81.

34. Gaharwar US, Meena R, Rajamani P. Iron oxide nanoparticles induced cytotoxicity, oxidative stress and DNA damage in lymphocytes. J Appl Toxicol JAT. 2017 Oct;37(10):1232-44.

35. Zhu M-T, Wang Y, Feng W-Y, Wang B, Wang M, Ouyang H, et al. Oxidative stress and apoptosis induced by iron oxide nanoparticles in cultured human umbilical endothelial cells. J Nanosci Nanotechnol. 2010 Dec;10(12):8584-90.

36. Zanganeh S, Hutter G, Spitler R, Lenkov O, Mahmoudi M, Shaw A, et al. Iron oxide nanoparticles inhibit tumour growth by inducing pro-inflammatory macrophage polarization in tumour tissues. Nat Nanotechnol. 2016 Nov;11(11):986-94.

37. Deiuliis JA, Kampfrath T, Zhong J, Oghumu S, Maiseyeu A, Chen LC, et al. Pulmonary T cell activation in response to chronic particulate air pollution. Am J Physiol Lung Cell Mol Physiol. 2012 Feb 15;302(4):L399-409.

38. Gu X-Y, Chu X, Zeng X-L, Bao H-R, Liu X-J Effects of PM2.5 exposure on the Notch signaling pathway and immune imbalance in chronic obstructive pulmonary disease. Environ Pollut Barking Essex 1987. 2017 Jul;226:163-73.

39. Castañeda AR, Pinkerton KE, Bein KJ, Magaña-Méndez A, Yang HT, Ashwood P, et al. Ambient particulate matter activates the aryl hydrocarbon receptor in dendritic cells and enhances Th17 polarization. Toxicol Lett. 2018 Aug;292:85-96.

40. Sun L, Fu J, Lin S-H, Sun J-L, Xia L, Lin C-H, et al. Particulate matter of $2.5 \mu \mathrm{m}$ or less in diameter disturbs the balance of $\mathrm{TH} 17 /$ regulatory $\mathrm{T}$ cells by targeting glutamate oxaloacetate transaminase 1 and hypoxia-inducible factor 1a in an asthma model. J Allergy Clin Immunol. 2020 Jan;145(1):40214.

41. Wan Q, Liu Z, Yang M, Wu J. Acceleratory effects of ambient fine particulate matter on the development and progression of atherosclerosis in apolipoprotein $\mathrm{E}$ knockout mice by downregulating CD4 + CD25 + Foxp3 + regulatory T cells. Toxicol Lett. 2019 Nov;316:27-34.

42. Brandenberger C, Rowley NL, Jackson-Humbles DN, Zhang Q, Bramble LA, Lewandowski RP, et al. Engineered silica nanoparticles act as adjuvants to enhance allergic airway disease in mice. Part Fibre Toxicol. 2013 Jul 1;10:26.

43. Phalen RF, Mannix RC, Drew RT. Inhalation exposure methodology. Environ Health Perspect. 1984 Jun;56:23-34. 
44. Wong BA. Inhalation exposure systems: design, methods and operation. Toxicol Pathol. 2007 Jan;35(1):3-14.

45. Wiśniewska A, Olszanecki R, Totoń-Żurańska J, Kuś K, Stachowicz A, Suski M, et al. AntiAtherosclerotic Action of Agmatine in ApoE-Knockout Mice. Int J Mol Sci. 2017 Aug 4;18(8).

46. Suski M, Kiepura A, Wiśniewska A, Kuś K, Skałkowska A, Stachyra K, et al. Anti-atherosclerotic action of GW9508 - Free fatty acid receptors activator - In apoE-knockout mice. Pharmacol Rep. 2019 Jul 1;71(4):551-5.

\section{Figures}

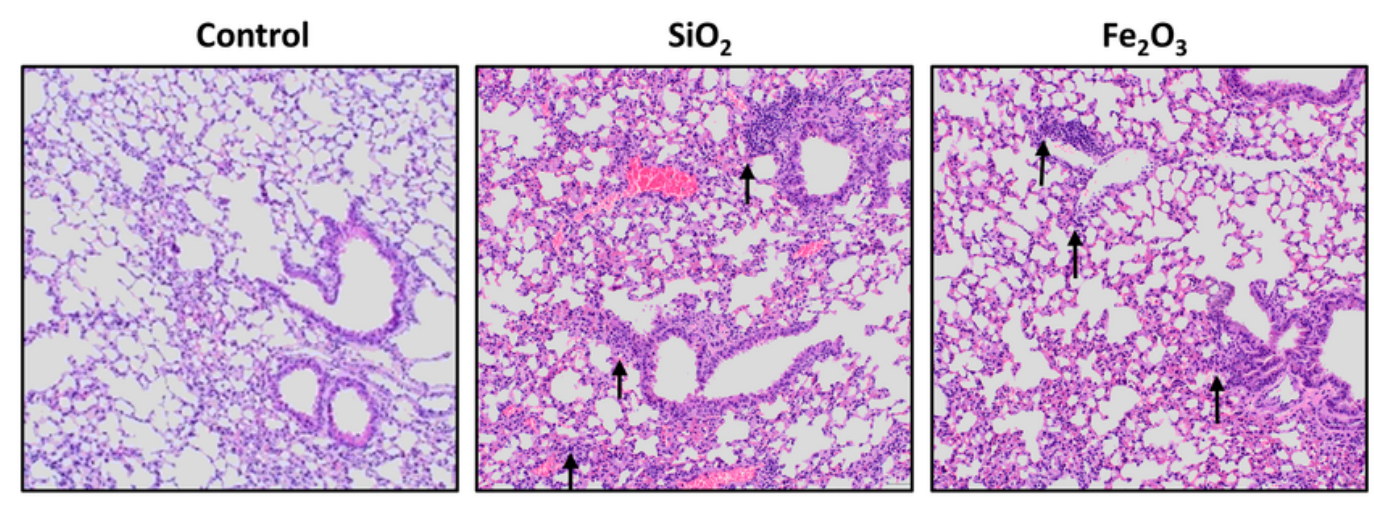

\section{Figure 1}

Effects of silica and ferric oxide nanoparticles on lung morphology. Representative pictures from HEstained lung sections showing accumulation of aggregates of inflammatory cells (lymphocytes, plasma 
cells) alongside the walls of minor respiratory tracts - in a pattern of pulmonary BALT hyperplasia (black arrows).

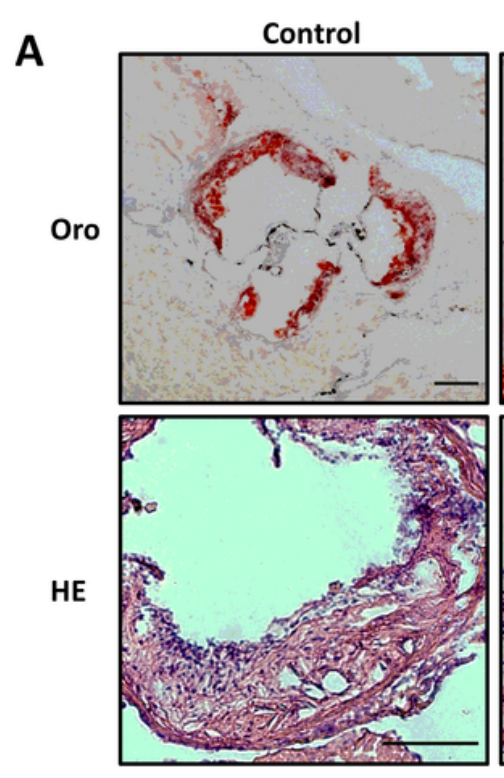

B

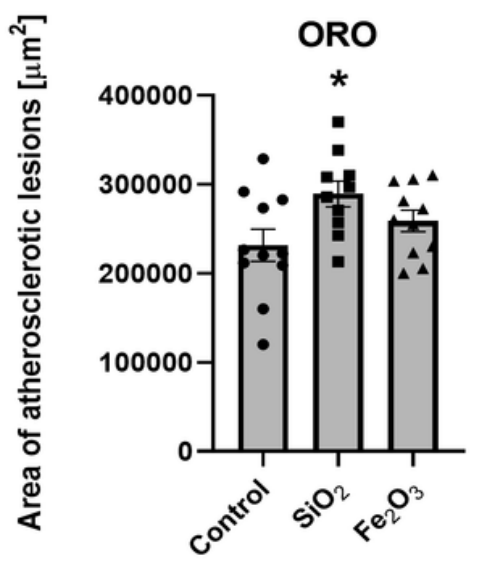

$\mathrm{SiO}_{2}$

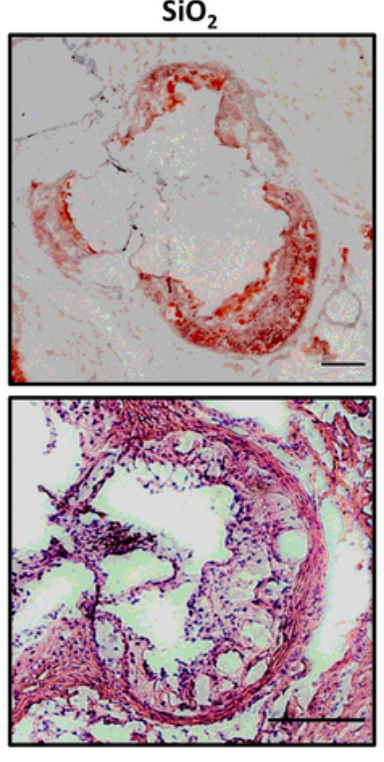

C

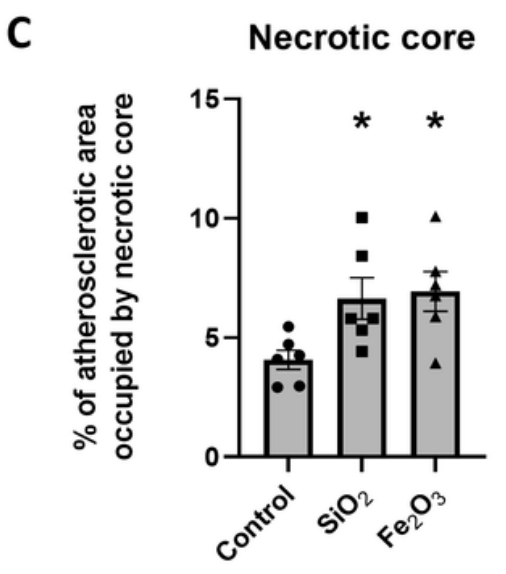

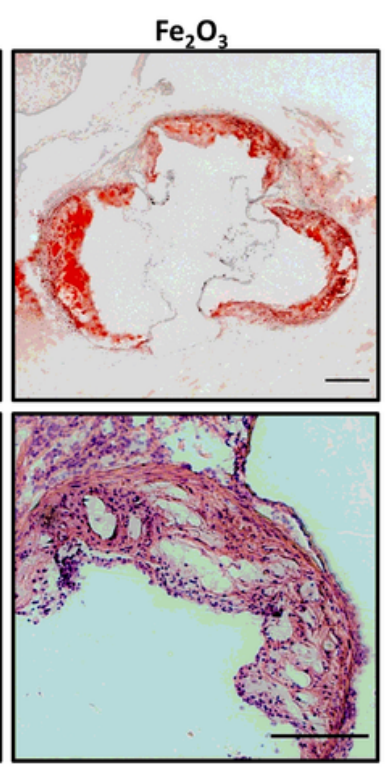

Necrotic core

Figure 2

Effects of silica and ferric oxide nanoparticles on atherosclerosis progression. Representative oil-red 0stained atherosclerotic lesions (scale bar $=200 \mu \mathrm{m}$ ) and HE-stained necrotic cores (scale bar $=50 \mu \mathrm{m}$ ) measured in aortic roots by the cross-section method in control, SiNPs-treated and FeNPs-treated mice (A). Quantitative analysis of areas of atherosclerotic plaques (B), $n=10-11$ per group. Quantitative analysis of areas of necrotic cores (C), $n=6$ per group. Each point represents a single mouse and bars show mean $\pm \mathrm{SEM} ;{ }^{*} \mathrm{p}<0.05$ as compared to the control. 

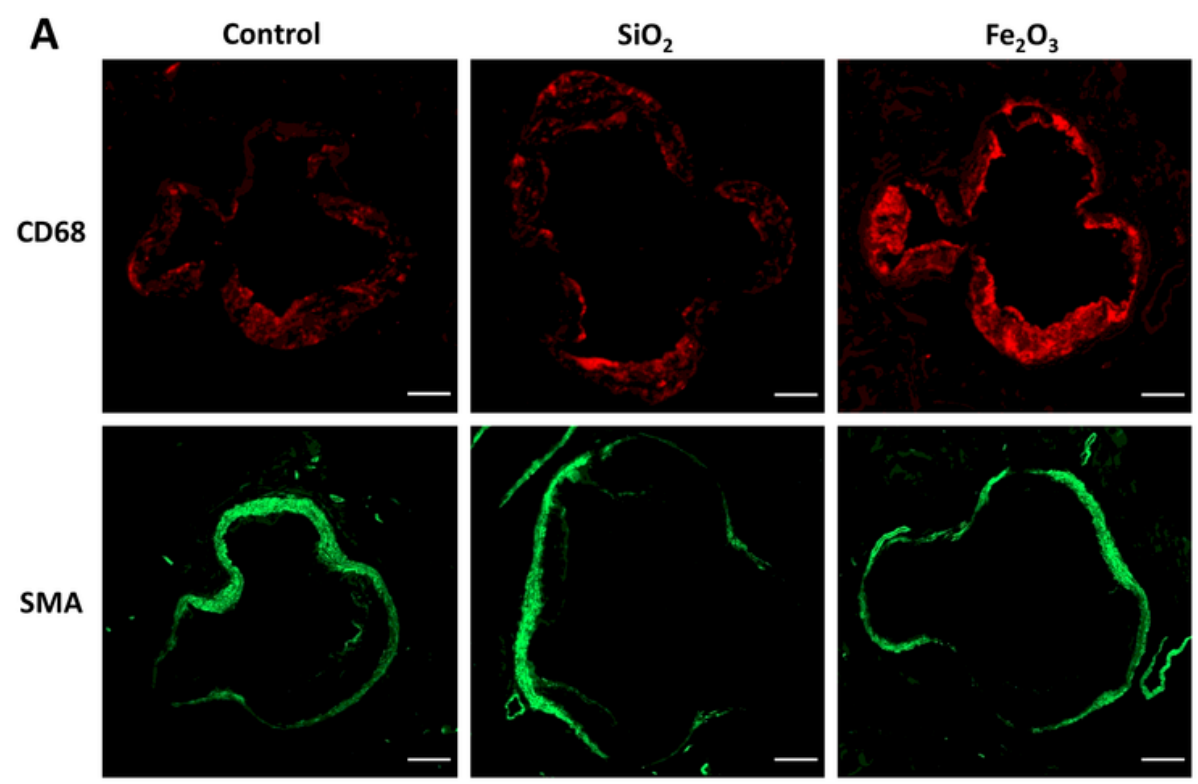

B
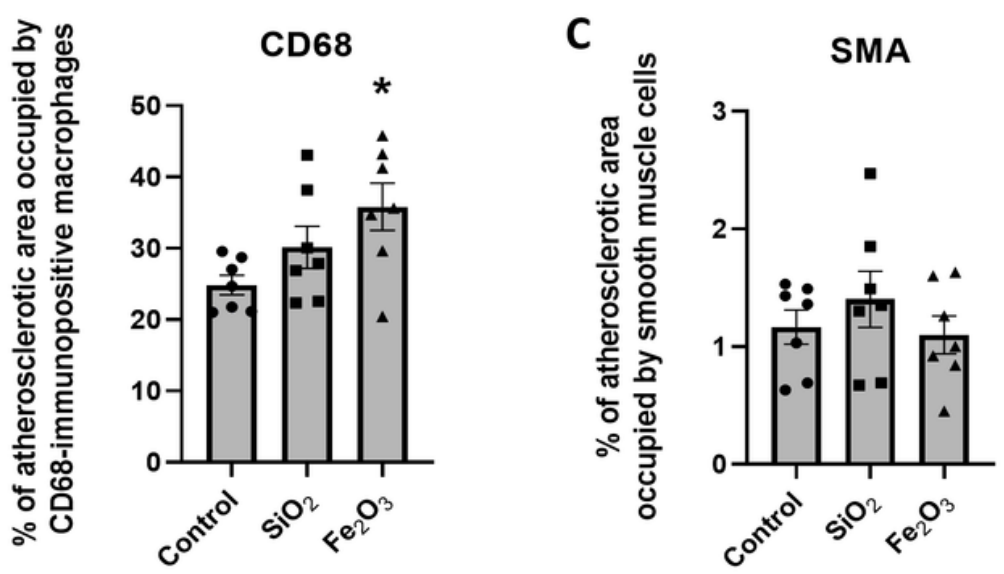

\section{Figure 3}

Influence of silica and ferric oxide nanoparticles on atherosclerotic plaques structure. Representative immunohistochemical staining of aortic roots $(A)$ with respective quantitative analysis of CD68-positive macrophages (B) and smooth muscle a-actin (SMA)-positive cells content (C) in control, SiNPs-treated and FeNPs-treated mice. Each point represents a single mouse and bars show mean \pm SEM; * $p<0.05$ as compared to the control; $\mathrm{n}=7$ per group; scale bar $=200 \mu \mathrm{m}$. 

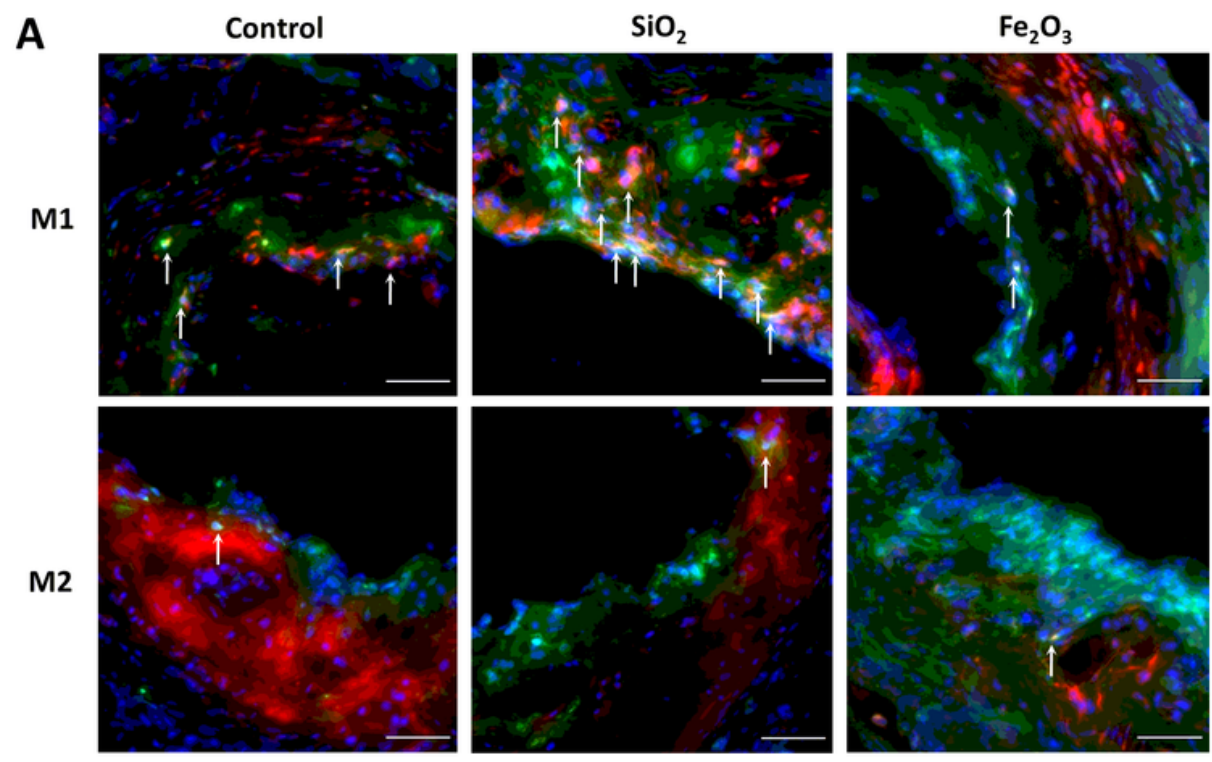

B

M1 macrophages

M2 macrophages
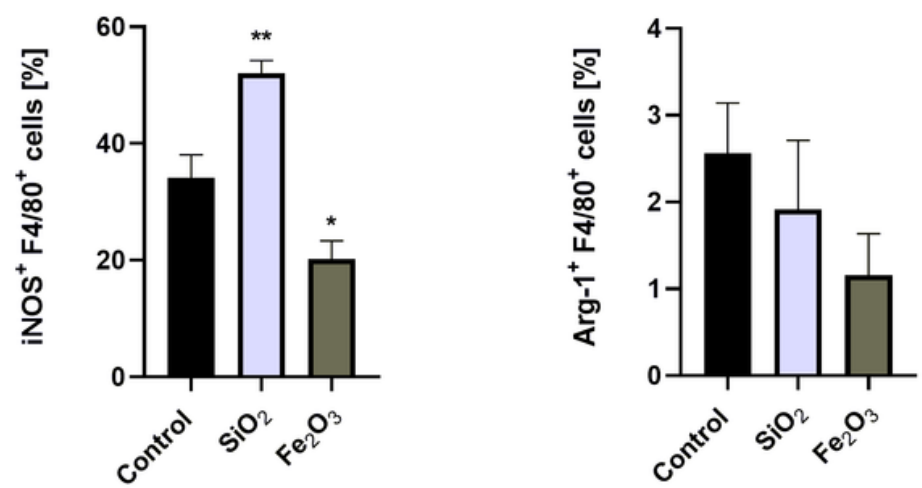

\section{Figure 4}

Macrophages phenotyping in atherosclerotic plaques in apoE-/- mice after exposure to nanoparticles.

Representative images of immunohistochemical-stained aortic roots showing F4/80 (green), nitric oxide synthase 2 (iNOS)/arginase 1 (Arg-1) (red) and Hoechst (blue) co-localization, respondent to macrophage phenotype (white arrows) in control, SiNPs-treated and FeNPs-treated mice; scale bar $=50 \mu \mathrm{m}(\mathrm{A})$. Graphs represent quantitative analysis of $\mathrm{M} 1$ and $\mathrm{M} 2$ macrophages in atherosclerotic plaques expressed as mean \pm SEM $(B) ; * p<0.05, * \star p<0.01$ as compared to control; $n=3-5$ per group. 

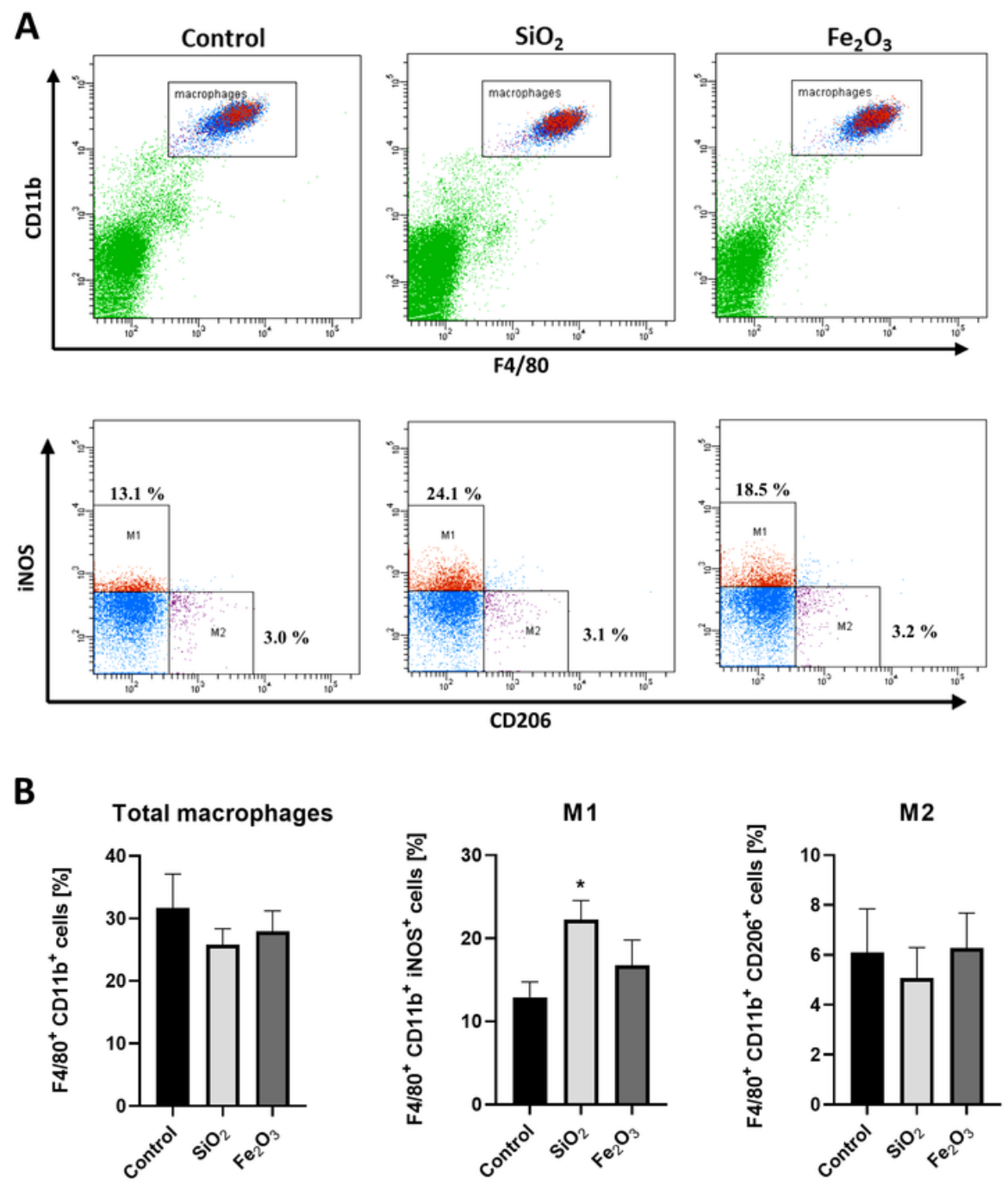

Figure 5

Flow cytometry analysis of peritoneal macrophages in apoE-/- mice. Gating strategy for CD11b+and F4/80+ cells (total macrophages) (A). Dot plots showing iNOS-positive cells (M1 macrophages) and CD206-positive cells (M2 macrophages) in control group, SiNPs-treated and FeNPs-treated mice (A). Quantification of M1, M2 and total macrophages in peritoneum (B). Data on the graphs presented as mean \pm SEM; $* p<0.05$ as compared to the control; $n=5-6$ per group. 
M1 markers
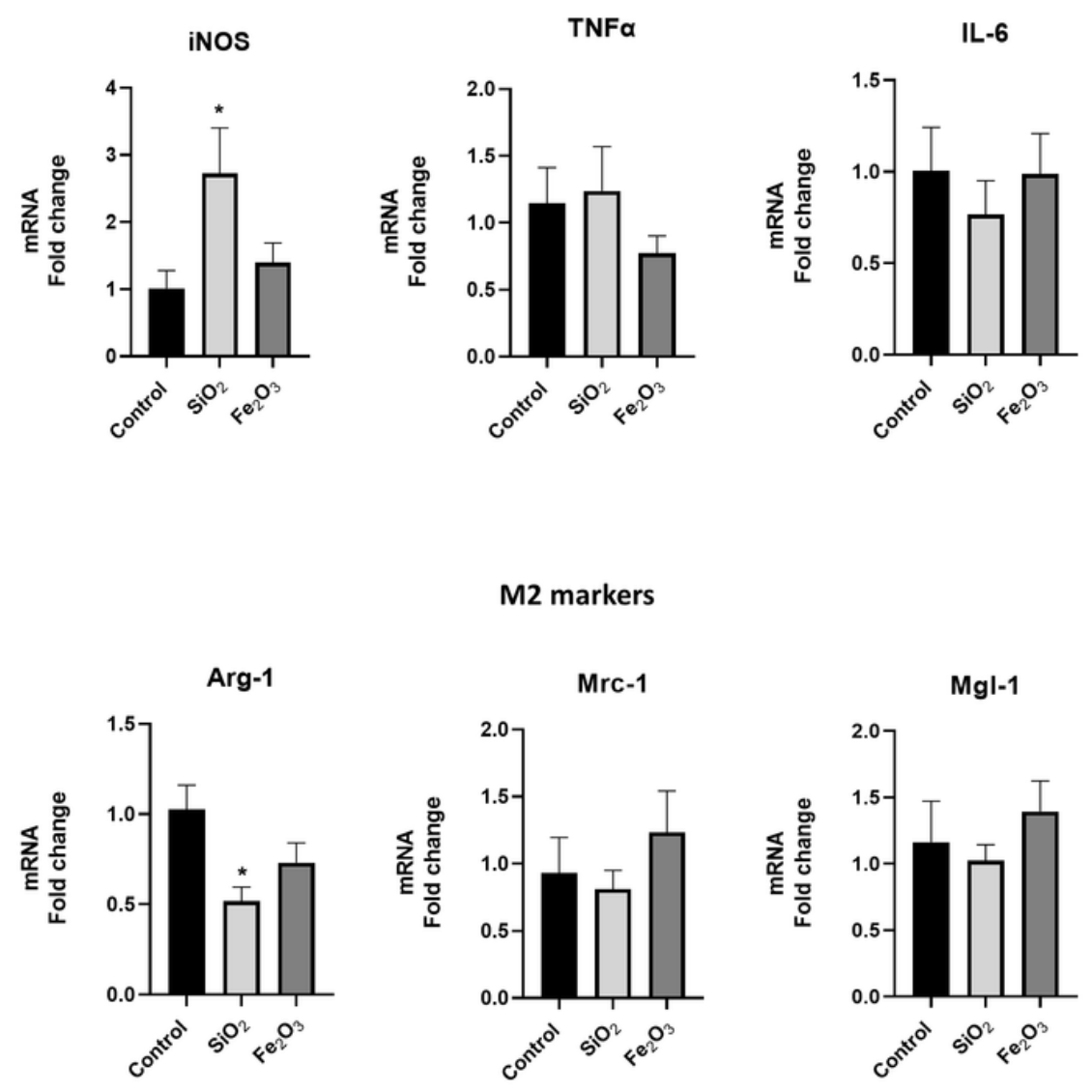

Figure 6

The expression of M1 and M2 makers in aortas of apoE-/- mice. Data presented as mean fold change \pm SEM; * $\mathrm{p}<0.05$ as compared to the control; $\mathrm{n}=4$ - 6 per group. 
A
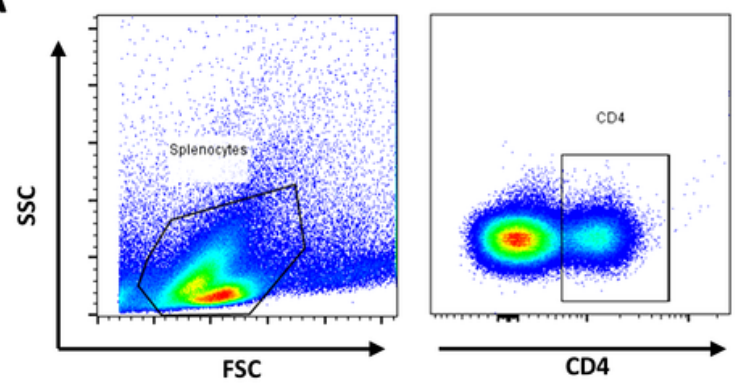

C

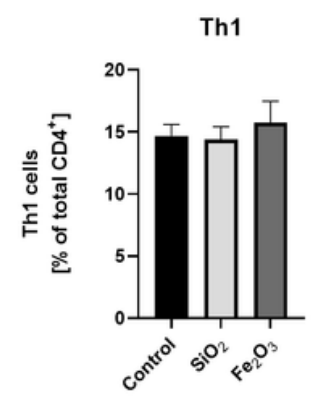

Th17

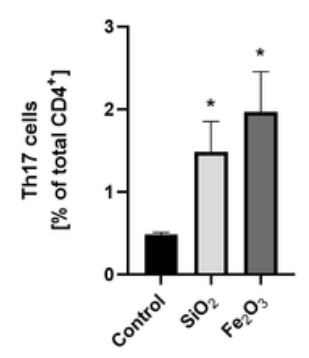

B

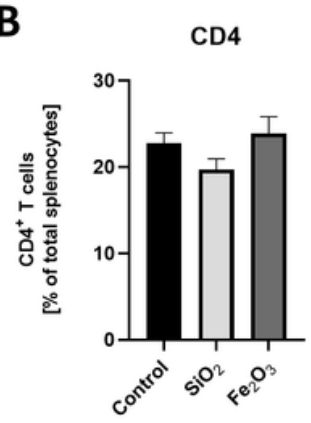

Th2
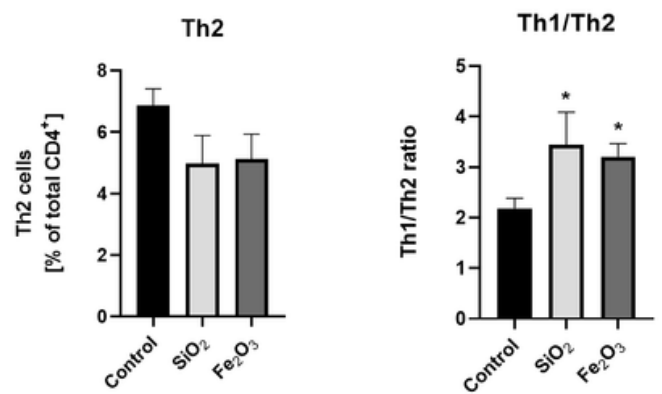

Treg

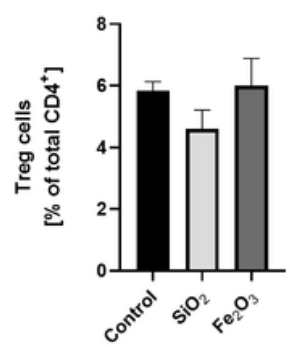

Th17/Treg

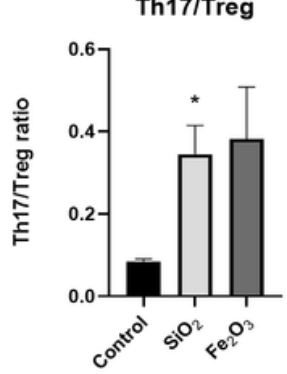

Figure 7

Flow cytometry analysis of splenic T lymphocytes in apoE-/- mice. Gating strategy for CD4+ T cells (A). Quantification of total CD4+ T lymphocytes in spleen (B). Percentage of respective subpopulations of lymphocytes T (Th1, Th2, Th17, Treg) and the ratio of Th1/Th2 and Th17/Treg (C). Data on the graphs presented as mean $\pm \mathrm{SEM} ;{ }^{*} \mathrm{p}<0.05$ as compared to the control; $\mathrm{n}=6$ per group.

\section{Supplementary Files}

This is a list of supplementary files associated with this preprint. Click to download.

- Graphicalabstract.tif 